\title{
Power Control in Wireless Sensor Networks with Variable Interference
}

\author{
Michele Chincoli, ${ }^{1}$ Aly Aamer Syed, ${ }^{2}$ Georgios Exarchakos, ${ }^{1}$ and Antonio Liotta ${ }^{1}$ \\ ${ }^{1}$ Electrical Engineering Department, Eindhoven University of Technology, De Zaale, 5612 AJ Eindhoven, Netherlands \\ ${ }^{2}$ NXP Semiconductors, High Tech Campus 60, 5656 AG Eindhoven, Netherlands
}

Correspondence should be addressed to Michele Chincoli; m.chincoli@tue.nl

Received 17 March 2016; Revised 20 May 2016; Accepted 8 June 2016

Academic Editor: Sergio F. Ochoa

Copyright (C) 2016 Michele Chincoli et al. This is an open access article distributed under the Creative Commons Attribution License, which permits unrestricted use, distribution, and reproduction in any medium, provided the original work is properly cited.

\begin{abstract}
Adaptive transmission power control schemes have been introduced in wireless sensor networks to adjust energy consumption under different network conditions. This is a crucial goal, given the constraints under which sensor communications operate. Power reduction may however have counterproductive effects to network performance. Yet, indiscriminate power boosting may detrimentally affect interference. We are interested in understanding the conditions under which coordinated power reduction may lead to better spectrum efficiency and interference mitigation and, thus, have beneficial effects on network performance. Through simulations, we analyze the performance of sensor nodes in an environment with variable interference. Then we study the relation between transmission power and communication efficiency, particularly in the context of Adaptive and Robust Topology (ART) control, showing how appropriate power reduction can benefit both energy and spectrum efficiency. We also identify critical limitations in ART, discussing the potential of more cooperative power control approaches.
\end{abstract}

\section{Introduction}

Wireless sensor networks (WSNs) are gaining significant attention from institutions, entrepreneurs, and researchers alike, due to the promising and innovative applications in the context of Internet of Things (IoT) and due to the lowering cost of the components, which enables a widespread deployment. Practical scenarios can be categorized in three main groups: rural, urban, and indoor. In rural environments, the sensor nodes are spread discontinuously in vast areas such as battlefields, natural sites, and farmlands, for the purpose of monitoring, surveying, and management. WSN deployments in urban scenarios denote the increasing demand for more efficient cities, which are increasingly expected to provide high-tech services to the citizens (commonly referred to as Smart City). Last category comprehends indoor scenarios and embraces wireless network installations in buildings (e.g., home, office, and shopping mall) [1]. Such areas may be smaller than in the previous cases, but, on the other hand, the density of the sensor nodes may be much higher. As for Smart Cities, also here it is very important to accurately monitor energy consumption, which helps both the consumers (to be aware of the expenses in real time) and the service provider (to estimate and predict the quantity of resources to provide, e.g., Smart Grids [2]). Home and Building Automation (HABA), as described in [3], will impact the current home architecture and design and even change people's habits. The skeleton of future buildings will see many wires replaced by wireless devices. Electrical household appliances can be collectively managed either by one remote control or by a smart phone. Blinds, heaters, and coolers can adapt autonomously to physical metrics (e.g., temperature, light level, and humidity) and predictable human presence for intelligent future applications [4].

Zigbee is one of the instances that are trying to standardise indoor connected, cooperative systems. Other examples are the WiFi HaLow, WirelessHART, and 6LoWPAN, to mention but a few. Sensor nodes are constrained devices, having low transmission power, battery, memory, and computational capacity $[5,6]$. WSNs can comprise a large and growing number of devices, becoming in some cases highly dense in HABA scenarios. The nodes can assume the role of routers, 
aggregators, and coordinators. Moreover, in the urban and indoor domains, the sensor nodes are typically combined with actuators, objects that react with specific actions when triggered [7]. The focus of our research lies on the study of indoor scenarios including HABA applications that are based on ZigBee specifications.

WSNs inherit typical problems of other wireless technologies while also having their own complications. The wireless channel is unreliable and variable over space, time, and frequency, providing nondeterministic signal strength $[8,9]$. Sensor nodes, within the HABA context, are in the majority of cases static or, at most, barely moved to different positions. Currently, indoor environments contain many WiFi networks, whose power is much higher than the power used by 802.15 .4 devices and Bluetooth communications.

Besides the influence of external interference, since sensor nodes may operate in very dense networks, the internal interference is also an important factor. When devices transmit at their highest transmission power, contention can heavily affect the performance of the whole network, in terms of QoS and energy consumption [10]. Simultaneous nearby communications at significant transmission power provoke packet collisions; then retransmissions and Clear Channel Assessment (CCA) fail, with increasing number of access attempts and backoff time [11]. Hence, the transceivers are overused, which causes further battery depletion, packet delay, and packet losses. It is the area where power control can play a crucial role.

The work presented hereafter builds on the preliminary findings introduced in [12]. We have significantly extended our analysis, studying a broader range of cases and the effects of node density and of a fine-grained transmission power variation. Situations with low, intermediate, and high interference have been studied in combination with small, medium, and strong transmission power levels. We show that increasing transmission power toward the maximum is not always a wise solution. At the same time, decreasing the transmission power down to the minimum is not a right solution either. Depending on the network condition, an intermediate transmission power should balance the communication quality of each link. We compare the performance of homogeneous transmission power control (TPC) with Adaptive and Robust Topology (ART) control, as introduced in [13] and further evaluated in [14], under the same conditions. We find that ART reduces the contention, while respecting the Packet Reception Ratio (PRR) constraint. However, our analysis unveils that, in its current formulation and due to lack of collaboration among nodes, ART still fails to meet the combined requirements of power efficiency and spectrum efficiency. Because of the large number of nodes and the complexity of WSNs, a flexible, distributed, and collaborative approach should be preferable, as we previously hinted in $[15,16]$.

\section{Transmission Power Control Protocols}

For many years, researchers have been targeting TPC protocols to solve the energy problem relating to good link quality $[13,14,17-26]$. The author of [10] considers a collection of works divided in homogeneous and heterogeneous transmission power configuration of the network components, highlighting why it is important that nodes transmit at different power levels, depending on specific criteria and circumstances. Yet, multihop communications allow signals to travel on a sequence of short distances, employing lower transmission power than direct communications. This contributes to lowering the total energy consumption and the overall contention in the network. Below, we present an overview of some of the most significant methods and elaborate on ART, which is used in our study.

Power Control with Blacklisting (PCBL) requires an initial beaconing phase where all the nodes broadcast a sequence of messages at every transmission power level, while their neighbors record the PRR [18]. Power levels are chosen depending on the required PRR for each link. Unreliable links, those which cannot achieve the required PRR at any transmission power, are blacklisted and are not subsequently used for communication.

In adaptive transmission power control (ATPC) the authors use a linear regression model to obtain the coefficients of the linear relationship and predict the appropriate transmission power, based on the desired Received Signal Strength Indicator (RSSI) threshold [17]. Their goal is to obtain the minimum distinguished transmission power per neighbor bases, while maintaining good link quality. Eventually, a routing table, containing the proper transmission power per link, is built.

On-Demand TPC (ODTPC) is designed to eliminate the bootstrapping phase required by algorithms like PCBL and ATPC, while reducing energy consumption and providing good link quality [19]. ODTPC is based on the assumption that there is a threshold in RSSI that separates good links from intermediate or bad links [27].

Adaptive On-Demand Transmission Power Control (AODTPC) [20] is an attempt to improve the performance of ODTPC under channel fading. While ODTPC reacts to changes in signal strength, AODTPC uses a Kalman filter to estimate future values of RSSI based on past measurements. The advantage of the Kalman filter over other prediction techniques is its lower amount of memory and computation required to store and process the variation of the analyzed variable. The authors show by simulations how the algorithm improves energy savings and node lifetime in comparison to ODTPC.

Hackmann et al. propose ART to reduce power consumption and channel contention in WSNs [13]. The authors choose to rely on packet reception rate (PRR) as link quality estimator, since both RSSI and the Link Quality Indicator (LQI) are demonstrated to be too sensitive to the environment. The study also provides a case in favor of TPC on a per-link basis as a way to reduce network contention in dense networks, demonstrating that lowering the transmission power under high contention can reduce interference and thus increase the average PRR.

In [21], two distributed local algorithms, LMA and LMN, are discussed. The transmission power is chosen based on the "life acknowledge messages," as defined in [21] that are received from the neighbors, which is compared with two 
thresholds. The goal is to improve the lifetime of WSNs while keeping the nodes connected.

Another algorithm to enhance the lifetime of the network is described in [22]. In this case, a technique to build a geographical topology is used. Links in Non-Line-of-Sight are excluded and the transmission power is calculated based on the Signal-to-Interference-and-Noise Ratio (SINR) and Received Signal Strength (RSS) targets.

Similarly, in [23] the transmission power is controlled by measuring RSSI to ensure good link quality based on the estimated and reference SINR values. Moreover the authors determine an adaptive Golden Receive Power Range (GRPR) to preserve the performance from the fading variations. Again, comparing RSSI with two thresholds (low and high) set in GRPR, the transmission power is either increased or decreased accordingly.

Energy efficiency is addressed also in [24] where the techniques Hybrid and AEWMA are proposed specifically for WSNs. The first, through a closed control loop that iterates on the available transmission powers, and the second, through calculations using the transmission power, received power and average noise, determine the transmission power level to use for a communication. The results show enhancements in energy savings of up to $57 \%$ compared to the case using a fixed power level.

The impact, in terms of energy consumption, of one node on the whole network is studied in [25], where it is suggested that the energy consumed by the whole network is of greater importance than the energy that a node itself consumes.

Authors in [26] introduce a TPC algorithm based on PRR in order to reduce the transmission power and provide sufficient data packet delivery, at the expense of frequent overhead injected in the network. The work is concluded emphasizing the importance of periodic updates of PRR estimation for PRR-based algorithms, although they require higher energy consumption proportional to the frequency of the updates.

A classification is performed instead in [28], in which transmission power control algorithms used for energy efficiency are divided into active and passive techniques. The former consider protocols that may be identified in one among the MAC, Network, or Transport layers. Each protocol aims to improve the energy consumption by adjusting the transmission power. Passive techniques manipulate hardware components (i.e., the radio transceiver) while a sensor node is supposed to either transmit, receive, or stay idle [29]. The authors emphasize the importance of QoS of real applications in TPC algorithms.

Compared to earlier work, our main contribution is to investigate the applicability of TPC in WSNs to mitigate interference, which results in communication improvements and, consequently, also in energy reduction. We change transmission power over time, aiming for the minimum whenever possible, by taking into account PRR requirements.

\section{ART Description}

Among the above discussed works, we chose to analyze ART for different reasons. Firstly, as it is asserted in [13, 30, 31],
RSSI is a good indicator only when PRR is very high. Otherwise, RSSI alone cannot provide reliable information. For this reason, we prefer to use PRR (as ART does) as direct link quality estimator (LQE). Secondly, ART considers contention reduction along with energy efficiency, although only PRR is analyzed as performance indicator in the network. Ultimately, the overhead of extra control messages is avoided calculating PRR at the transmitter side, exploiting Acknowledgements (ACKs) reception.

ART is a PRR-based TPC algorithm implemented at the transmitter side and can be used to reduce energy consumption and contention. It works on a per-link basis. Considering node pairs, one transmitter and one receiver, a stream of data is transmitted. The packets have fixed size and are generated periodically at an interarrival time $\mu$. The transmission power, $P_{\text {tx }}$, can assume different values distributed in discrete levels provided in the radio transceiver. When a packet is correctly received, an ACK is sent back for confirmation. PRR is calculated as the ratio of the number of received packets over transmitted packets, within a window $W$ of $N$ transmitted packets. In this case, the number of received packets is considered equal to the number of received ACKs. Then, PRR is compared with two thresholds, $\eta_{l}$ and $\eta_{h}$, chosen in order to keep it in the specified range of values. $\eta_{l}$ in [13] is calculated as the product of the window size and PRR target $p\left(\eta_{l}=N * p\right)$. Similarly $\eta_{h}=N * p^{\prime}$, where $p^{\prime}$ represents PRR upper bound as the maximum acceptable performance by the system. Tuning $P_{\mathrm{tx}}$ to the lowest power, the failures should be kept under the threshold $1-\eta_{l}$. Following the experiments of the authors in [13], because of the bimodal relationship between PRR and $P_{\mathrm{tx}}$, decreasing the power may lead to a PRR lower than $p$. For this reason, the authors have introduced a trial state, following a reduction of power level, to evaluate the effects on the PRR. In this case, if the number of failures in $W$ is higher than $\left(1-\eta_{h}\right) \cdot N$, the calculation of PRR is stopped, the previous $P_{\mathrm{tx}}$ is restored, and $W$ is flushed. Otherwise, if the trial is successful, the new power is confirmed. Moreover, ART protocol provides a gradient-based mechanism to monitor the contention on a link, which is enabled when PRR is lower than $\eta_{l}$. Thus, if the contention is low, $P_{\mathrm{tx}}$ is increased; otherwise $P_{\mathrm{tx}}$ is decreased. In our implementation, we have disabled this mechanism, in order to analyze the outcome in preferring high transmission powers. Figure 1 shows the Finite State Machine (FSM) of our examined ART version. If PRR is greater than $\eta_{h}, P_{\mathrm{tx}}$ is decreased by one level; otherwise, if PRR is lower than $\eta_{l}, P_{\mathrm{tx}}$ is raised by one level. In any other case, $P_{\mathrm{tx}}$ stays constant. After the comparison, $W$ is flushed and the calculation starts again. Algorithm 1 provides a pseudocode specification of ART.

\section{Evaluation}

4.1. Experimental Setup. Simulations are used to investigate a broad range of scenarios to achieve statistical significance and repeatability while having more control over the networks' behavior. In our setup, we monitor the performance of a pair of sensor nodes (used as probes) placed in the center of a variable grid of other sensor nodes, used as interferers (Figure 2). Every cell of the grid contains a point-to-point 


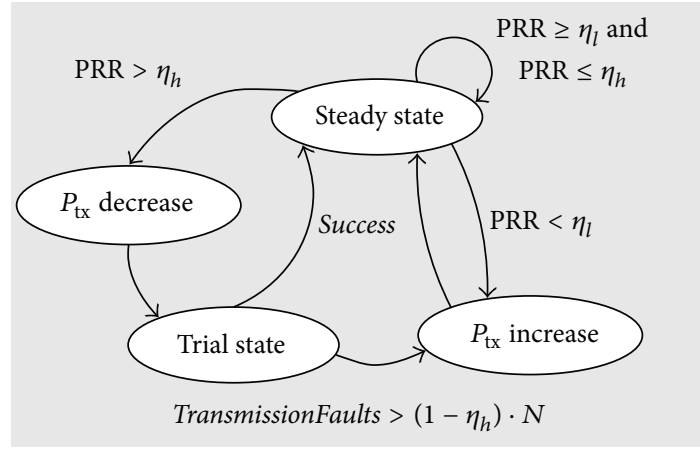

FIgURE 1: Finite State Machine of the protocol ART.

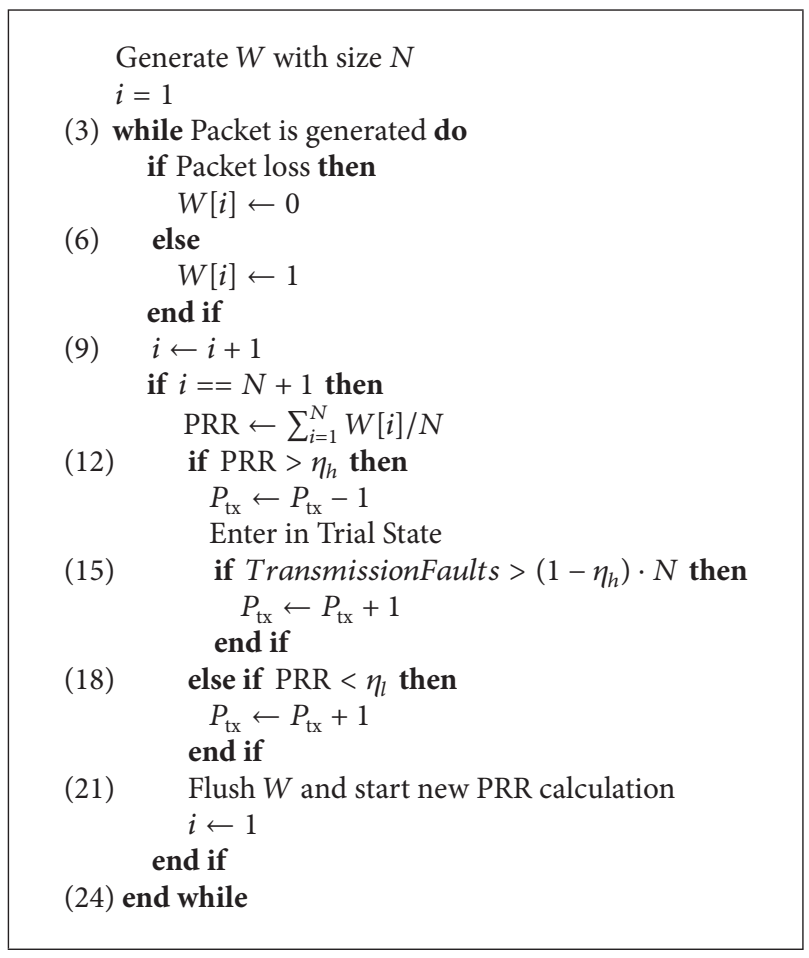

Algorithm 1: ART pseudocode specification.

communication between one transmitter and one receiver. The relative distances between adjacent transmitters are indicated as $\Delta x$ and $\Delta y$, respectively. The relative distance within transmitter-receiver pairs, $d$, is set to five meters, which provides a good overall experimental tradeoff. The experiment (simulation) parameters and settings are summarized in Table 1.

We modeled transceivers with a sensitivity $S$ of $-95 \mathrm{dBm}$ and 22 different transmission power levels $\left(P_{\mathrm{tx}}^{\text {(lev) }}\right)$ comprised between $-35 \mathrm{dBm}$ and $10 \mathrm{dBm}$. The default transmission power is $0 \mathrm{dBm}$. The transmission of the ACK packets is not involved in the TPC process, so their transmission power is set to the value specified at that instant. The PHY and MAC are based on the IEEE 802.15.4 standard and the radio transceiver operates at the $2.45 \mathrm{GHz}$ frequency, employing Direct Sequence Spread Spectrum (DSSS) and
TABLE 1: Experimental setup for simulations.

\begin{tabular}{|c|c|c|}
\hline Parameter & Symbol & Value \\
\hline Sensitivity & $S$ & $-95 \mathrm{dBm}$ \\
\hline Packet interarrival time & $\mu$ & $100 \mathrm{~ms}$ \\
\hline Packet size & $s$ & 50 bytes \\
\hline Transmission power levels & $P_{\mathrm{tx}}^{(\text {lev })}$ & 22 \\
\hline Number of retransmissions & & 3 \\
\hline Number of CCA attempts & & 4 \\
\hline Transmitter buffer size & & $\infty$ \\
\hline Grid horizontal gap & $\Delta x$ & $5-300 \mathrm{~m}$ \\
\hline Grid vertical gap & $\Delta y$ & $5-300 \mathrm{~m}$ \\
\hline Distance within transmitter-receiver pairs & $d$ & $5 \mathrm{~m}$ \\
\hline PRR window & $W$ & 100 \\
\hline PRR moving factor & $W^{\mathrm{mov}}$ & 100 \\
\hline Minimum transmission power & MinPow & $-35 \mathrm{dBm}$ \\
\hline Intermediate transmission power & InterPow & $-18.42 \mathrm{dBm}$ \\
\hline Default transmission power & DefPow & $0 \mathrm{dBm}$ \\
\hline Maximum transmission power & MaxPow & $10 \mathrm{dBm}$ \\
\hline \multirow[t]{3}{*}{ Network interferer density } & LowDense & 2 pairs \\
\hline & MidDense & 16 pairs \\
\hline & HighDense & 36 pairs \\
\hline \multicolumn{3}{|l|}{ ART } \\
\hline Low threshold & $\eta_{l}$ & $95 \%$ \\
\hline High threshold & $\eta_{h}$ & $99 \%$ \\
\hline Transmission power scale & & $3.18 \mathrm{dBm}$ \\
\hline Initial transmission power & & $0 \mathrm{dBm}$ \\
\hline
\end{tabular}

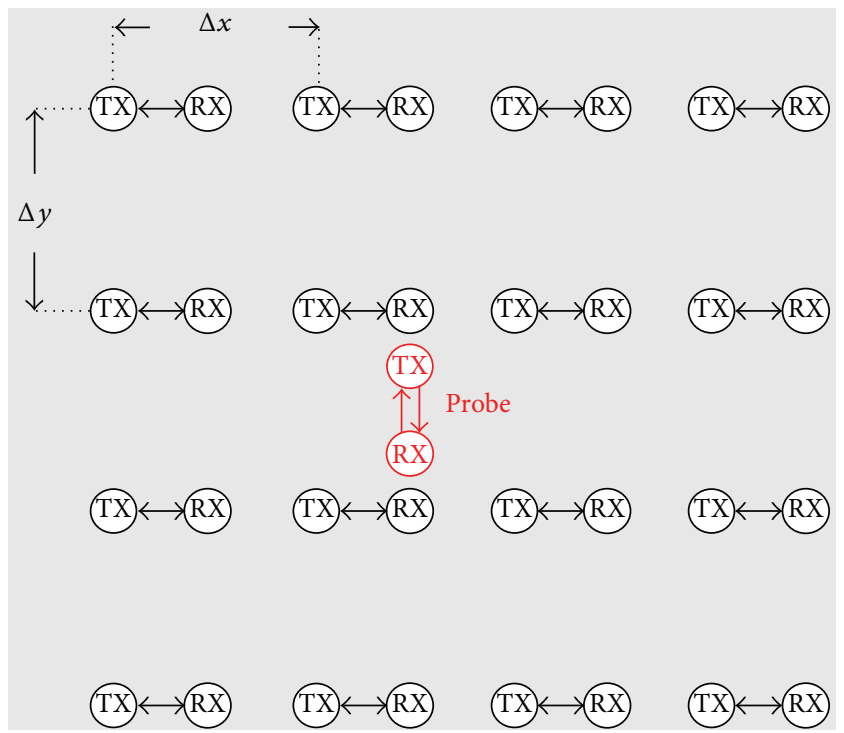

FIGURE 2: Simulation scenario including two sets of node pairs: one probe (in the middle) and sixteen interferers.

O-QPSK modulation. The simulations are carried out using the NS3 simulator: an open source, scalable, modular, and event-driven simulator, using the module Low Rate-Wireless Personal Area Network (LR-WPAN) from the release ns-3.23. Having a fully implemented reliable model for WSNs, several models for multiple aspects (i.e., path loss, fading, WiFi 
interference, and energy), and an active community, among the other features described above, NS3 simulator has been our favorite choice. In this study we have used exclusively the 802.15.4 module, which follows the 2006 version of the standard [11]. We adopted the site-general ITU-R P.1238-7 model in an office environment for large-scale path loss and the Nakagami model for small-scale fading [32]. The antennas were assumed to be ideally isotropic. We considered the unslotted Carrier Sense Multiple Access/Collision Avoidance (CSMA/CA) channel access technique and CCA mode 1, that is, energy above threshold. The maximum number of retries and CCA attempts is set to the default indicated by the standard 802.15.4, equal to three and four, respectively. The transmitter buffer size of the generated packets was set to infinite in order to capture only the packet loss incurred by the CCA fails and collisions.

We consider two sets of simulations. In the first set, we explore three different scenarios: LowDense, MidDense, and HighDense, whereby the number of pairs in the grid is equal to 2,16 , and 36 , respectively. Counting also the monitored couple, the network contains a total of 6, 34, and 74 nodes, correspondingly (an exemplary grid with 16 couples is shown in Figure 2). For each scenario, only one homogeneous transmission power is used in the network: the minimum (MinPow), the intermediate (IntPow, $-18.42 \mathrm{dBm}$ ), and the default transmission power (DefPow). In the second set of simulations, the number of pairs is fixed to 16 and two cases are compared: when ART is enabled and when the constant default transmission power is used. In the former case, the initial transmission power is equal to $0 \mathrm{dBm}$. All transmitters generate traffic following a Poisson distribution with an average interarrival time $\mu$ of $100 \mathrm{~ms}$ and a payload size $s$ of 50 bytes. $\Delta x$ and $\Delta y$ are varied from 5 to 300 meters; thus the grid can be either stretched or shrunk. In this set of simulations, the intensity of interference is modified by changing the transmission power and the distance of the transmitters in the grid from the probe pair.

The generation and independence of streams of pseudorandom numbers in NS3 can be done in two different ways, either through seed or through number of runs. In the first case, $1.8 \times 10^{19}$ independent streams can be generated, whereas, in the second one, $2.3 \times 10^{15}$ independent substreams can be extracted using the same seed. We use substreams to get random independent input to test each individual scenario. In this case, the period of the simulations must be shorter than $7.6 \times 10^{22}$ random numbers, the period of a pseudorandom subsequence. In each scenario, an amount of around 10,000 packets is generated. The randomness is given by the Nakagami fading model [32], which provides an additive channel loss to the signal and the packet generation. Since the sequence of random numbers is lower than the subsequence period, we use the number of runs as variable. For a specific scenario, we collect the results from 10 simulations with different number of runs and average them for statistical significance.

Assuming the absence of any external interference, we analyze the PRR, latency, number of CCA attempts, and retransmissions of the probe pair. PRR is computed as the

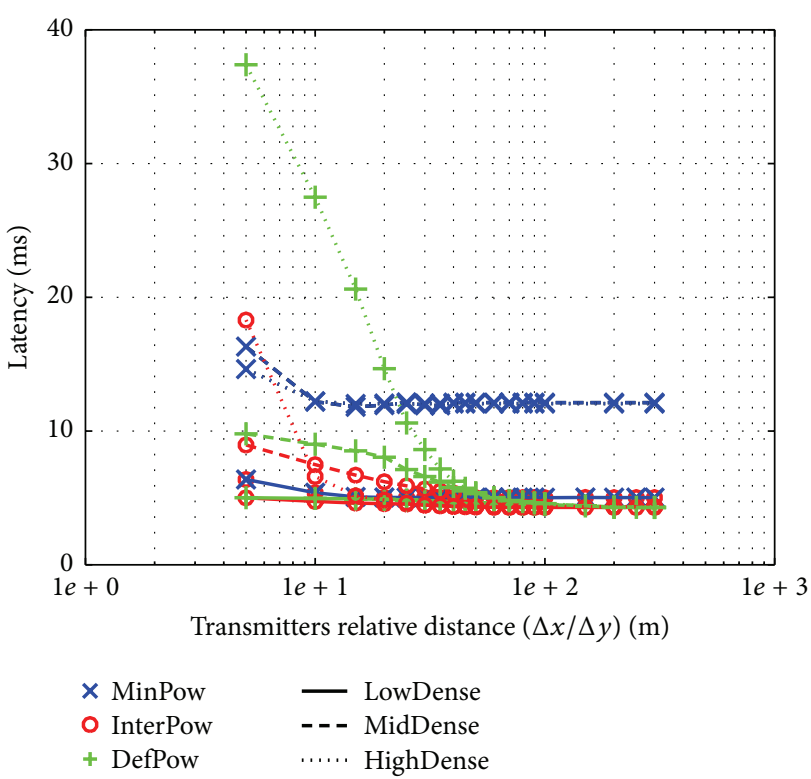

FIgURE 3: Average latency of the probe pair's traffic using MinPow, InterPow, and DefPow in LowDense, MidDense, and HighDense scenarios.

number of received ACK messages divided by the number of transmitted packets and the latency as the time interval between the transmission of a message and reception of the related ACK. Latency is only computed for successful transmissions, as it only makes sense when a message is delivered. PRR is calculated in a window of 100 packets.

4.2. Results Using Homogeneous Transmission Power. Here we discuss the effect of varying the transmission power levels and number of nodes in the network. We use the probe pair to measure this effect as described above. The transmission power is kept constant during each simulation scenario and is the same for all the nodes. We compare the performance of the reference couple for the scenarios LowDense, MidDense, and HighDense, described above, in the three cases of different transmission power MinPow, InterPow, and DefPow, while varying $\Delta x$ and $\Delta y$ (the distance between the transmitters in the grid).

In Figures 3-6 we present the results, whereby each point is a combination of some of the variable parameters mentioned before. Thus, the level of interference is variable too. For example, we have the lowest interference possible combining LowDense, MinPow, and long distance for $\Delta x$ and $\Delta y$, while we have the highest interference when HighDense and DefPow are used with small $\Delta x$ and $\Delta y$. The interference is referred to the probe pair placed in the middle of the grid. The main idea is to assess the effect of the transmission power in the three different scenarios at different distances. For instance, using very low transmission power, the interference may be detrimental to the monitored nodes. Indeed in Figure 6 the number of CCA attempts is very low in the case of MinPow, below 0.5 and equal to 0 starting from 15 meters, regardless of the node density. At the same time, the number 


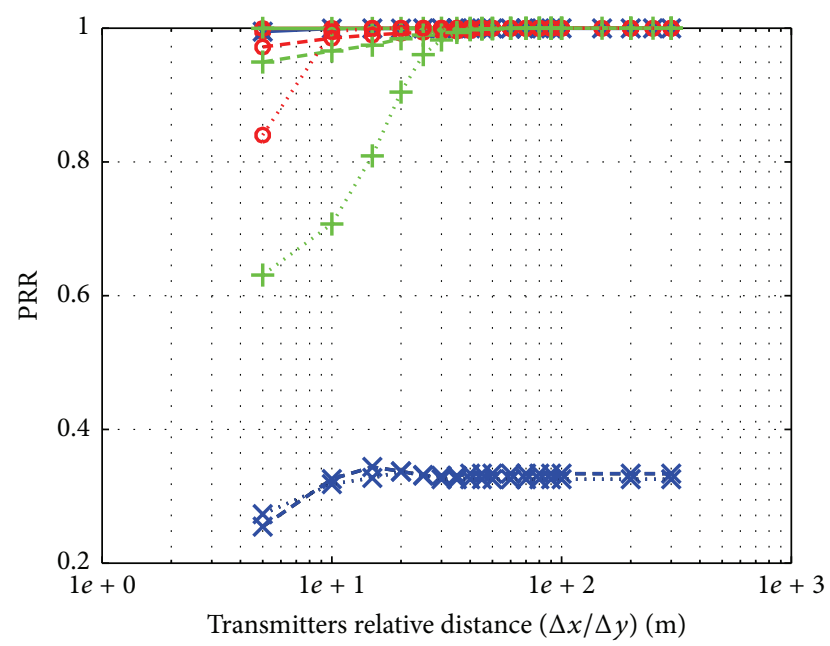

$\begin{array}{lll}\times \text { MinPow } & - & \text { LowDense } \\ \text { O InterPow } & --- & \text { MidDense } \\ \text { + DefPow } & \text {..... } & \text { HighDense }\end{array}$

FIgURE 4: Average packet reception rate of the probe pair's traffic using MinPow, InterPow, and DefPow in LowDense, MidDense, and HighDense scenarios.

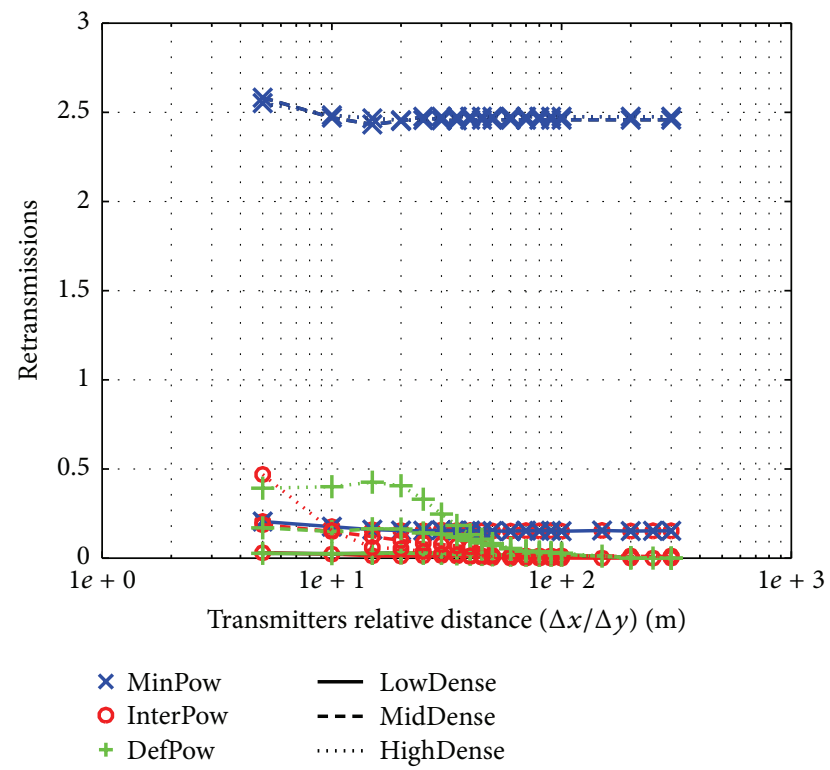

FIGURE 5: Average number of retransmissions of the probe pair's traffic using MinPow, InterPow, and DefPow in LowDense, MidDense, and HighDense scenarios.

of retransmissions in Figure 5 is very high, around 2.5, with MidDense and HighDense, and low in the case of LowDense. The effect is that, in the former situation, PRR is significantly low around 30\% from 10 meters and beyond, and the latency is high, close to $12 \mathrm{~ms}$. While, in the second situation, the latency is $6.5 \mathrm{~ms}$ at $5 \mathrm{~m}$ of distance flattening to $5 \mathrm{~ms}$ and PRR is over $99.5 \%$. Thus, MinPow is a good choice when the interference is weak; otherwise the communications suffer massively the contention.

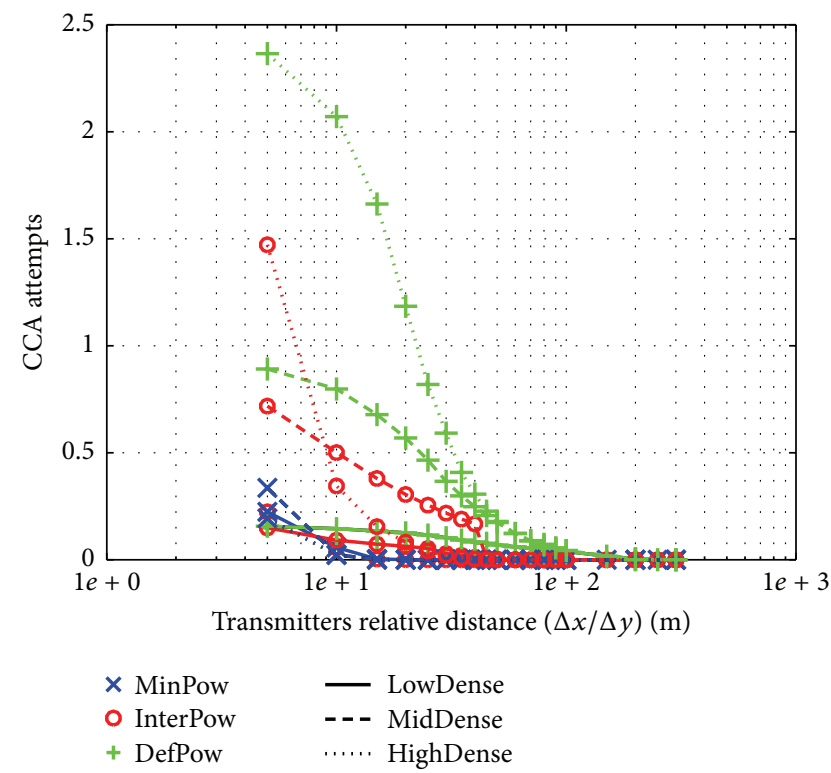

FIGURE 6: Average number of CCA attempts of the probe pair's transmitter using MinPow, InterPow, and DefPow in LowDense, MidDense, and HighDense scenarios.

The case of DefPow (i.e., $P_{\mathrm{tx}}=0 \mathrm{dBm}$ ) is different. The worst case scenario happens when many nodes transmit in a dense network (HighDense) where the number of CCA attempts (Figure 6) is as high as 2.3 when $\Delta x$ and $\Delta y$ are equal to the minimum, decreasing asymptotically until reaching no contention at 200 meters. Instead when the grid is less populated with nodes, the number of CCA attempts, when the grid is shrunk, is smaller than in the previous case more than one unit in MidDense and more than two units in LowDense. All the three curves meet in zero CCA attempts from 200-meter distance. As expected, the number of retransmissions is highest in HighDense but lower than 0.5 . Instead, looking at one value of the distance for $\Delta x$ and $\Delta y$ in Figure 5 the number of retransmissions diminishes as less nodes are in the grid. The performance shown in Figures 3 and 4 provides the answer to our claim in this paper. The latency is at its maximum value at $37.5 \mathrm{~ms}$ in the case of HighDense, which is reduced to 10 and $5 \mathrm{~ms}$ for the other two scenarios MidDense and LowDense. As the distance between the couple goes up, the latency decays consequently. Similarly the PRR is $63.1 \%, 94.94 \%$, and $99.98 \%$ for HighDense, MidDense, and LowDense, respectively, while $\Delta x$ and $\Delta y$ are equal to the minimum, increasing up to $100 \%$ at 100 meters for all the curves.

Lastly, using the intermediate transmission power, we obtain better results than in the previous cases. Compared to DefPow, the latency and the number of retransmissions and CCA attempts are lower and the PRR is higher, starting with a difference of $20 \%$ and $25 \%$ in the HighDense scenario at a distance of 5 and 10 meters. While compared in MidDense, PRR is higher, as much as $2 \%$ at the beginning, until the values in the two scenarios are the same when $\Delta x$ is equal to 80 meters. In Figure 3, it is possible to notice the big 
difference between the two curves, InterPow and DefPow, especially in HighDense, where the latency differs by about $19 \mathrm{~ms}$, increasing to $21 \mathrm{~ms}$, and finally reduces following the $x$-axis to the right. Then, although the difference between the two curves in LowDense and MidDense is smaller, InterPow outperformes DefPow in all the scenarios.

Looking at Figures 4 and 3, InterPow is also better than (i.e., MidDense and HighDense) or at least comparable (i.e., LowDense) to MinPow.

The key point of our results is that neither increasing nor decreasing too much the transmission power can provide good performance in the network. But instead, the network should adaptively find its best intermediate $P_{\mathrm{tx}}$ in order to reach a global optimum. Moreover we show that increasing the number of nodes, so increasing the amount of traffic in the network, produces more interference and, in turn, the performance of the network worsens. Some solutions are needed to provide reliability to a network in terms of connectivity and Quality of Service (QoS) guarantees. Next, we analyze the behavior of ART in one of the scenarios depicted above.

4.3. Results Using ART. The ART protocol is tested in a network composed of a grid of 16 interfere couples (Figure 2) and one probe pair in the middle. Each node runs ART starting at a transmission power $P_{\mathrm{tx}}$ set to $0 \mathrm{dBm}$. The results are compared with the ones obtained using a homogeneous transmission power as in DefPow. The thresholds are equal to $95 \%$ and $99 \%$ for the low and high threshold, respectively. The step size to switch from a level of transmission power to another is equal to 20 .

In Figure 7 we can see the average transmission power used during the simulations as outcome of ART. In the two closest positions (i.e., 5 and 10 meters) ART pushes the transmitter to use the maximum transmission power equal to $10 \mathrm{dBm}$. This happens because PRR, using the initial transmission power, does not satisfy the requirements to settle between the thresholds. Following ART policy, $P_{\mathrm{tx}}$ is increased. Intuitively, we see that the initial transmission power is an important factor for this purpose. Based on our previous results, the intermediate power level performs better than high $P_{\mathrm{tx}}$. So, setting the initial transmission power to a low value may result in a longer transition time before reaching the right power but, at the same time, may perform better using lower power levels. When $\Delta x$ and $\Delta y$ are lowered to 15 meters, we start obtaining benefits in terms of power savings, as the average transmission power is equal to about $-24 \mathrm{dBm}$. It finally stabilizes to $-29 \mathrm{dBm}$ as the couples of nodes are moved apart. Therefore the number of CCA attempts is lower than in DefPow (Figure 11) but the number of retransmissions is higher (Figure 10).

In view of the fact that ART's target is to keep PRR between the thresholds, we can notice in Figure 9 that indeed PRR is around $98 \%$ from a distance of 15 meters, while it was not at the shortest distance (94.2\%). Although PRR values in ART are lower than DefPow, they have been forced by the algorithm to be in the range $\eta_{l}-\eta_{h}$ and so evaluated acceptable for our setup, gaining transmission power reduction. On

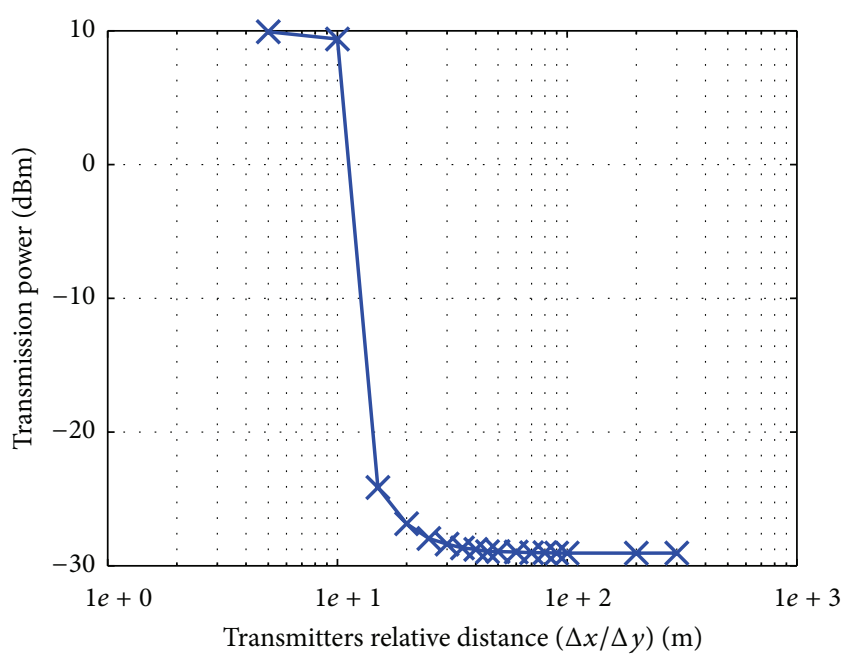

FIGURE 7: Average transmission power of the probe pair's transmitter using ART.

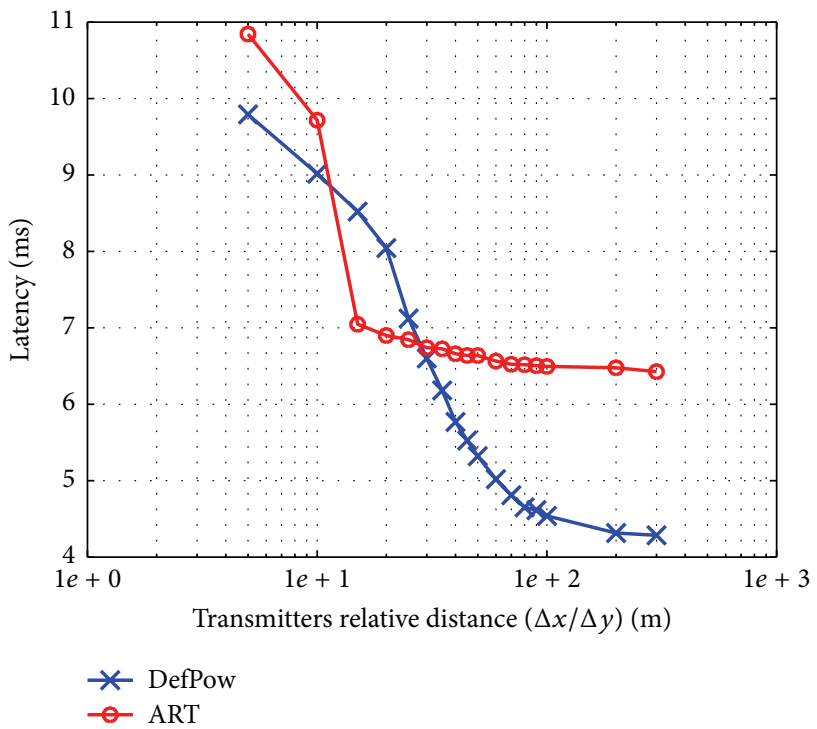

FIGURE 8: Average latency of the probe pair's traffic using ART and the default transmission power.

the contrary, the latency, which does not have any protocol constraints, is influenced indirectly by the PRR trend. In Figure 8 there are some points in which ART operates better than DefPow (i.e., from 15 to 25 meters). Instead, for instance, from 35 meters of distance, the latency in ART is higher and decreases more slowly than DefPow, reaching around $6.4 \mathrm{~ms}$ (against the $4.2 \mathrm{~ms}$ in DefPow) at the maximum distance.

\section{Conclusions}

Through simulations, we have verified our assumptions on the beneficial effects of transmission power reduction on network performance. Firstly, we showed that varying level of interference, represented by the load of nodes and traffic in the network, an arbitrary intermediate power level 


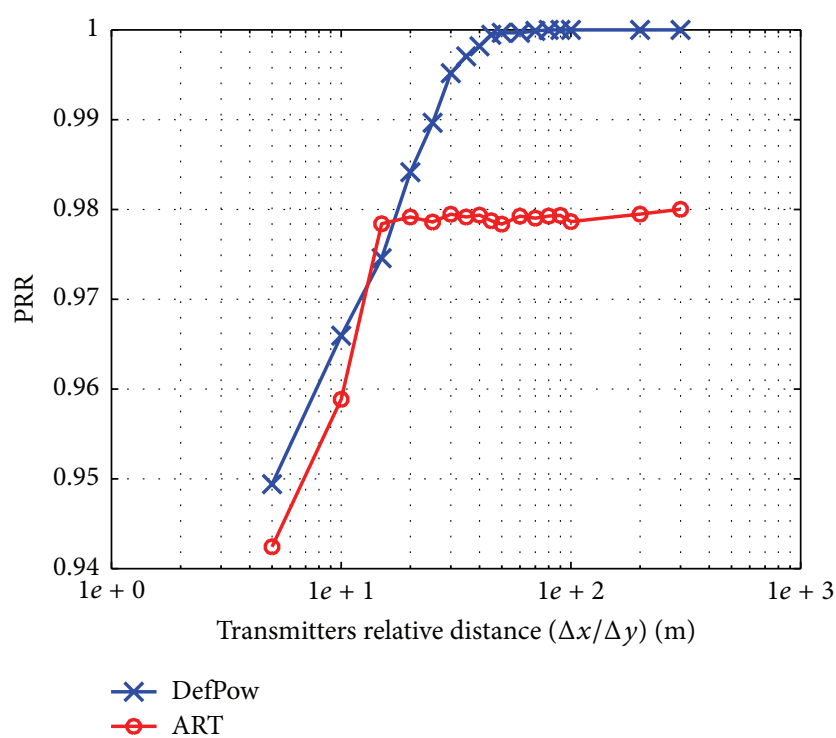

Figure 9: Average packet reception rate of the probe pair's traffic using ART and the default transmission power.

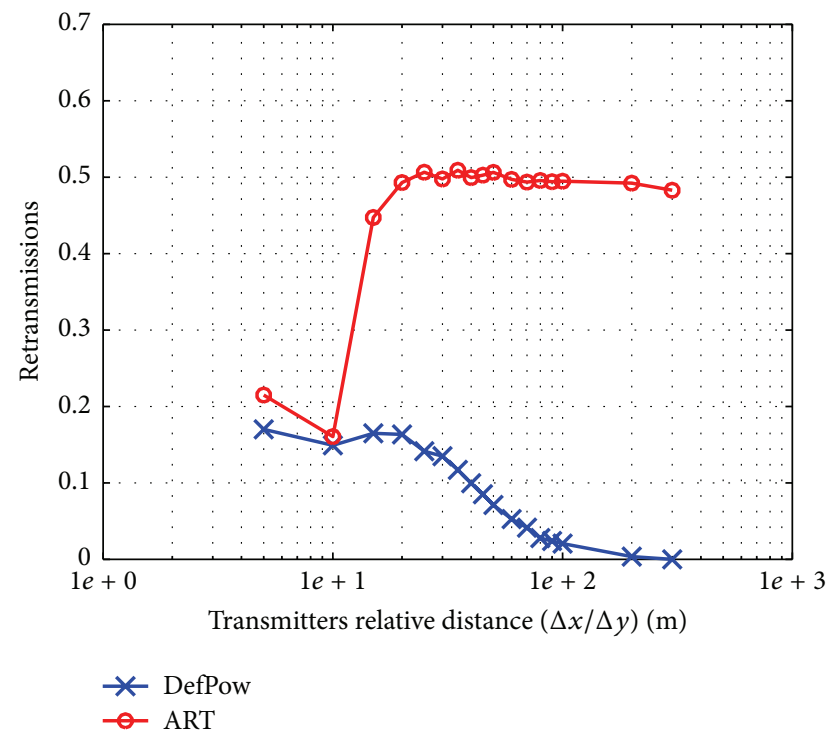

FIGURE 10: Average number of retransmissions of the probe pair's traffic using ART and the default transmission power.

outperforms the lowest and close to the highest transmission powers. This verifies that, under certain conditions, increasing transmission power does not necessarily lead to better performance. In fact, by lowering transmission power it is possible to reach an intermediate level that maximizes the overall network performance.

Intuitively, one would want a control protocol that keeps the nodes at minimum power to simultaneously pursue energy and spectrum efficiency. However, finding the bottom-line power which avoids loosing connectivity is not straightforward. That is why we believe that a promising research direction is to look at protocols that are smart [16] rather than deterministic [9].

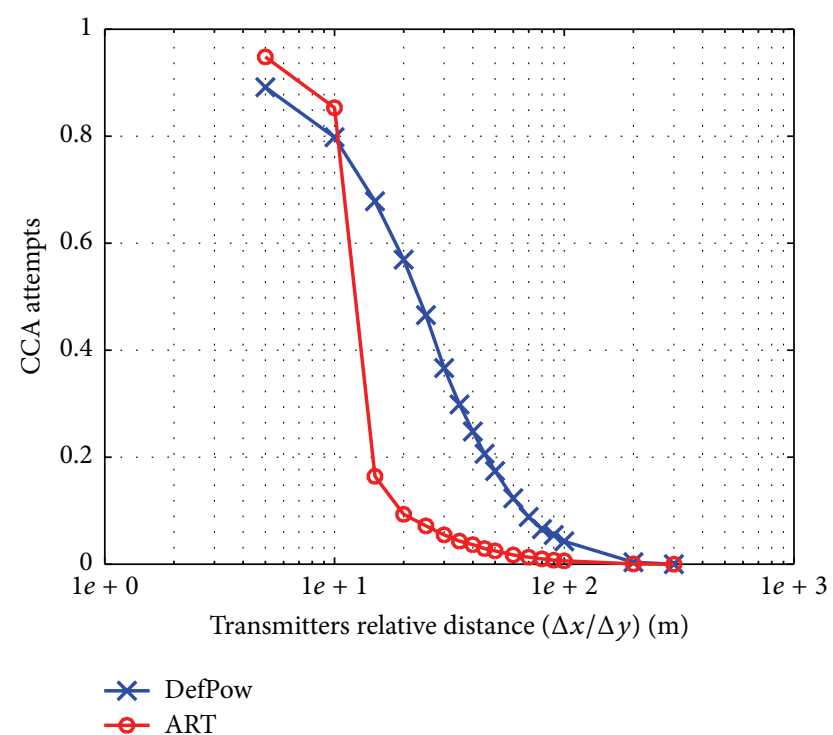

FIGURE 11: Average number of CCA attempts of the probe pair's transmitter using ART and the default transmission power.

We also observed that, compared to the situation in which the transmission power was always set to the default $0 \mathrm{dBm}$, ART achieved lower transmission power and maintained PRR in the required range. On the other hand, there are still many aspects to take into consideration to improve the algorithm. For example, ART does not consider latency requirements, nor does it fulfill them; thus it cannot guarantee QoS. The initial transmission power is relevant to the transition time used to research the right power. Plus, when PRR is not guaranteed, a lower transmission power may be more beneficial than just increasing by one or more levels as indeed is taken into account in the original version of ART through a flag related to the level of contention [13].

Moreover, the step size of transmission power levels is another variable to examine. On the one hand, a small gap provides more power levels options, which may help finding the best tradeoffs between energy and spectrum efficiency. On the other hand, greater granularity may lead to slow transients. Instability is another issue linked with power changes.

A major limitation of current adaptive protocols such as ART is their lack of collaboration among nodes. ART operates only on a per-link basis and the transmission power is set considering only the information gathered from the associated receiver. Collaborative, smart protocols have the potential to lead to the most significant breakthroughs and this is the direction we are going to explore next.

A promising direction is to explore self-learning methods and algorithms that may be implemented directly in the nodes, with the aim of allowing the sensor network to gradually determine the actions that lead to any given goal. Particularly, distributed, collaborative methods have the potential to use localized, in-node information towards global goals. Our first move will be to verify performance, convergence, and stability of learning-based methods and implement a pilot prototype. 


\section{Competing Interests}

The authors declare that there is no conflict of interests regarding the publication of this paper.

\section{Acknowledgments}

This work was jointly supported by NXP Semiconductors and Eindhoven University of Technology within the IMPULS program and by the EU INTER-IoT project (Grant 687283).

\section{References}

[1] G. Fortino, A. Guerrieri, G. M. P. O’Hare, and A. Ruzzelli, "A flexible building management framework based on wireless sensor and actuator networks," Journal of Network and Computer Applications, vol. 35, no. 6, pp. 1934-1952, 2012.

[2] A. Liotta, D. Geelen, G. van Kempen, and F. van Hoogstraten, "A survey on networks for smart-metering systems," International Journal of Pervasive Computing and Communications, vol. 8, no. 1, pp. 23-52, 2012.

[3] C. Gomez and J. Paradells, "Wireless home automation networks: a survey of architectures and technologies," IEEE Communications Magazine, vol. 48, no. 6, pp. 92-101, 2010.

[4] D. C. Mocanu, H. Bou Ammar, D. Lowet et al., "Factored four way conditional restricted Boltzmann machines for activity recognition," Pattern Recognition Letters, vol. 66, pp. 100-108, 2015.

[5] I. F. Akyildiz, W. Su, Y. Sankarasubramaniam, and E. Cayirci, "Wireless sensor networks: a survey," Computer Networks, vol. 38, no. 4, pp. 393-422, 2002.

[6] J. Yick, B. Mukherjee, and D. Ghosal, "Wireless sensor network survey," Computer Networks, vol. 52, no. 12, pp. 2292-2330, 2008.

[7] I. F. Akyildiz and I. H. Kasimoglu, "Wireless sensor and actor networks: research challenges," Ad Hoc Networks, vol. 2, no. 4, pp. 351-367, 2004.

[8] T. S. Rappaport, Wireless Communications: Principles and Practice, Prentice Hall, New York, NY, USA, 2002.

[9] A. Liotta, "Farewell to deterministic networks," in Proceedings of the 19th IEEE Symposium on Communications and Vehicular Technology in the Benelux (SCVT '12), pp. 1-4, IEEE, Eindhoven, The Netherlands, November 2012.

[10] P. Santi, "Topology control in wireless ad hoc and sensor networks," ACM Computing Surveys (CSUR), vol. 37, no. 2, pp. 164-194, 2005.

[11] IEEE Computer Society, LAN/MAN Standards Committee, Institute of Electrical and Electronics Engineers, and IEEE-SA Standards Board, IEEE Standard for Information Technology Telecommunications and Information Exchange between Systems-Local and Metropolitan Area NetworksSpecific Requirements. Part 15.4, Part 15.4, Institute of Electrical and Electronics Engineers, New York, NY, USA, 2006.

[12] M. Chincoli, C. Bacchiani, A. A. Syed, G. Exarchakos, and A. Liotta, "Interference mitigation through adaptive power control in wireless sensor networks," in Proceedings of the IEEE International Conference on Systems, Man, and Cybernetics (SMC '15), pp. 1303-1308, IEEE, Hong Kong, October 2015.

[13] G. Hackmann, O. Chipara, and C. Lu, "Robust topology control for indoor wireless sensor networks," in Proceedings of the 6th ACM Conference on Embedded Network Sensor Systems (SenSys '08), pp. 57-70, ACM, November 2008.
[14] Y. Fu, M. Sha, G. Hackmann, and C. Lu, "Practical control of transmission power for wireless sensor networks," in Proceedings of the 20th IEEE International Conference on Network Protocols (ICNP '12), pp. 1-10, IEEE, Austin, Tex, USA, November 2012.

[15] S. Galzarano, C. Savaglio, A. Liotta, and G. Fortino, "Gossipingbased AODV for wireless sensor networks," in Proceedings of the IEEE International Conference on Systems, Man, and Cybernetics (SMC '13), pp. 26-31, IEEE, Manchester, UK, October 2013.

[16] A. Liotta, "The cognitive NET is coming," IEEE Spectrum, vol. 50, no. 8, pp. 26-31, 2013.

[17] S. Lin, J. Zhang, G. Zhou, L. Gu, J. A. Stankovic, and T. He, "ATPC: adaptive transmission power control for wireless sensor networks," in Proceedings of the 4th International Conference on Embedded Networked Sensor Systems (SenSys '06), pp. 223-236, ACM, November 2006

[18] D. Son, B. Krishnamachari, and J. Heidemann, "Experimental study of the effects of transmission power control and blacklisting in wireless sensor networks," in Proceedings of the 1st Annual IEEE Communications Society Conference on Sensor and Ad Hoc Communications and Networks (SECON '04), pp. 289298, IEEE, Santa Clara, Calif, USA, October 2004.

[19] J. Kim, S. Chang, and Y. Kwon, "ODTPC: on-demand transmission power control for wireless sensor networks," in Proceedings of the International Conference on Information Networking (ICOIN '08), pp. 1-5, IEEE, Busan, South Korea, January 2008.

[20] M. M. Y. Masood, G. Ahmed, and N. M. Khan, "A Kalman filter based adaptive on demand transmission power control (AODTPC) algorithm for wireless sensor networks," in Proceedings of the International Conference on Emerging Technologies (ICET '12), pp. 1-6, IEEE, Islamabad, Pakistan, October 2012.

[21] M. Kubisch, H. Karl, A. Wolisz, L. C. Zhong, and J. Rabaey, "Distributed algorithms for transmission power control in wireless sensor networks," in Proceedings of the Wireless Communications and Networking (WCNC '03), pp. 558-563, IEEE, New Orleans, La, USA, March 2003.

[22] J.-H. You, J.-H. Jeon, T.-H. Lee, and Y.-G. Kwon, “Transmission power controlled localization with topology building for NLOS environments," International Journal of Distributed Sensor Networks, vol. 2014, Article ID 250692, 6 pages, 2014.

[23] W. Ikram, S. Petersen, P. Orten, and N. F. Thornhill, "Adaptive multi-channel transmission power control for industrial wireless instrumentation," IEEE Transactions on Industrial Informatics, vol. 10, no. 2, pp. 978-990, 2014.

[24] L. H. A. Correia, D. F. Macedo, A. L. dos Santos, A. A. F. Loureiro, and J. M. S. Nogueira, "Transmission power control techniques for wireless sensor networks," Computer Networks, vol. 51, no. 17, pp. 4765-4779, 2007.

[25] L. Xu, D. T. Delaney, G. M. P. Ohare, and R. Collier, "The impact of transmission power control in wireless sensor networks," in Proceedings of the 12th IEEE International Symposium on Network Computing and Applications (NCA '13), pp. 255-258, IEEE, Cambridge, Mass, USA, August 2013.

[26] M. Nikodem, M. Słabicki, T. Surmacz, and B. Wojciechowski, "Transmission power control based on packet reception rate," in Proceedings of the 6th IEEE International Conference on New Technologies, Mobility and Security (NTMS '14), pp. 1-4, Dubai, UAE, April 2014.

[27] M. Zuniga, I. Irzynska, J.-H. Hauer, T. Voigt, C. A. Boano, and K. Roemer, "Link quality ranking: getting the best out of unreliable links," in Proceedings of the 7th IEEE International Conference on 
Distributed Computing in Sensor Systems (DCOSS '11), pp. 1-8, IEEE, Barcelona, Spain, June 2011.

[28] N. A. Pantazis and D. D. Vergados, "A survey on power control issues in wireless sensor networks," IEEE Communications Surveys and Tutorials, vol. 9, no. 4, pp. 86-107, 2007.

[29] S. Galzarano, A. Liotta, and G. Fortino, "QL-MAC: a Q-learning based MAC for wireless sensor networks," in Algorithms and Architectures for Parallel Processing, R. Aversa, J. Kołodziej, J. Zhang, F. Amato, and G. Fortino, Eds., vol. 8286 of Lecture Notes in Computer Science, pp. 267-275, Springer, Berlin, Germany, 2013.

[30] N. Baccour, A. Koubaa, L. Mottola et al., "Radio link quality estimation in wireless sensor networks: a survey," ACM Transactions on Sensor Networks (TOSN), vol. 8, no. 4, article 34, 2012.

[31] K. Srinivasan, P. Dutta, A. Tavakoli, and P. Levis, "Understanding the causes of packet delivery success and failure in dense wireless sensor networks," in Proceedings of the 4th International Conference on Embedded Networked Sensor Systems (SenSys '06), pp. 419-420, ACM, Boulder, Colo, USA, November 2006.

[32] M. Nakagami, "The m-distribution-a general formula of intensity distribution of rapid fading," in Statistical Method of Radio Propagation, Pergamon, Oxford, UK, 1960. 

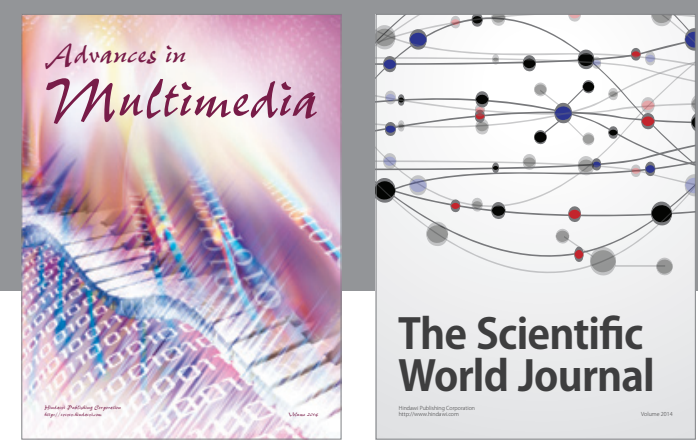

The Scientific World Journal
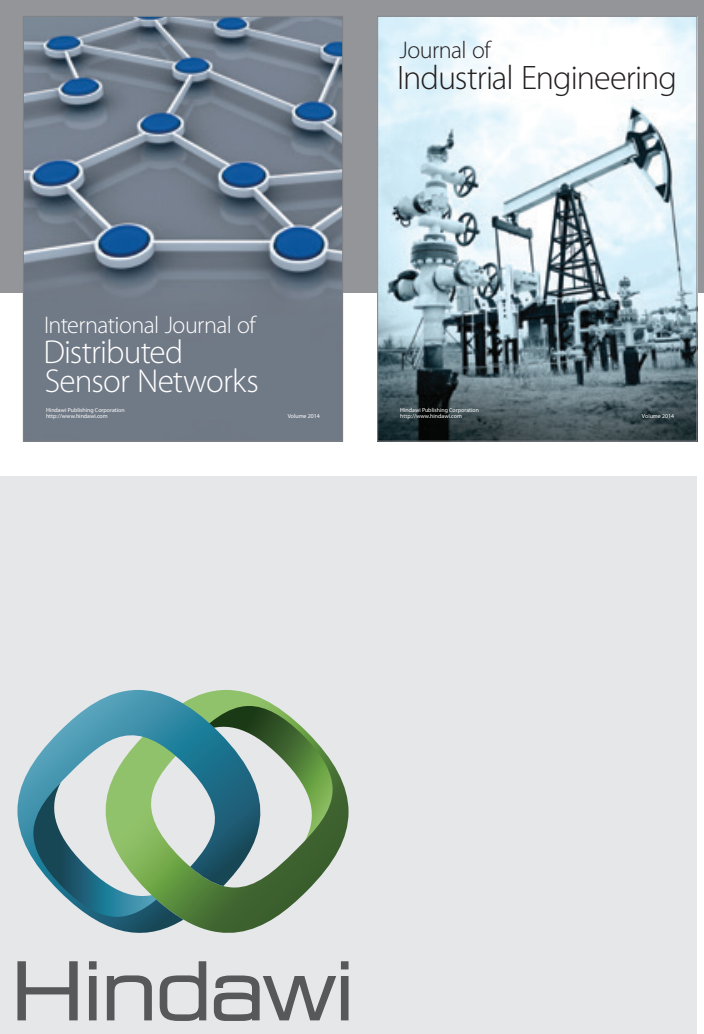

Submit your manuscripts at

http://www.hindawi.com

\section{Computer Networks} and Communications
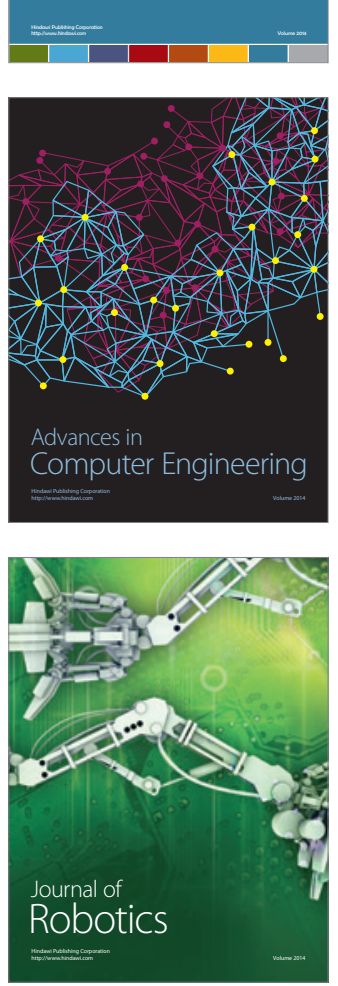
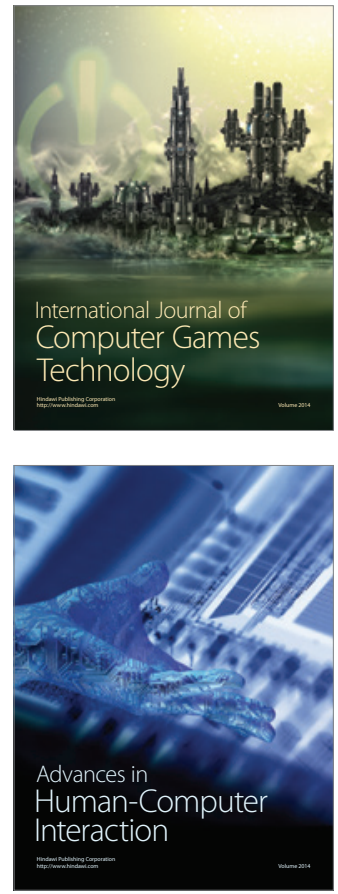
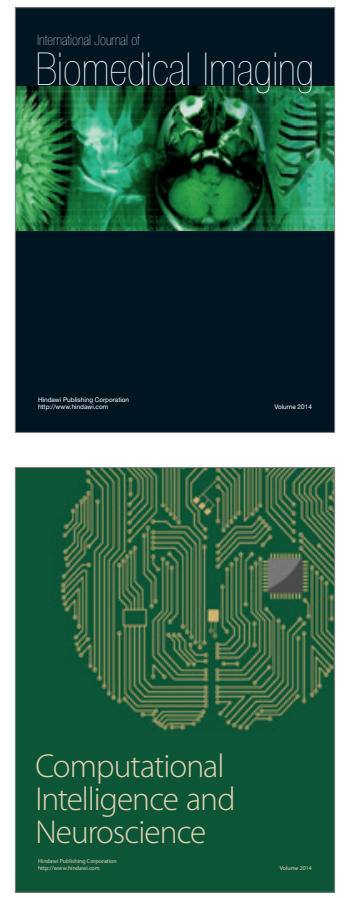
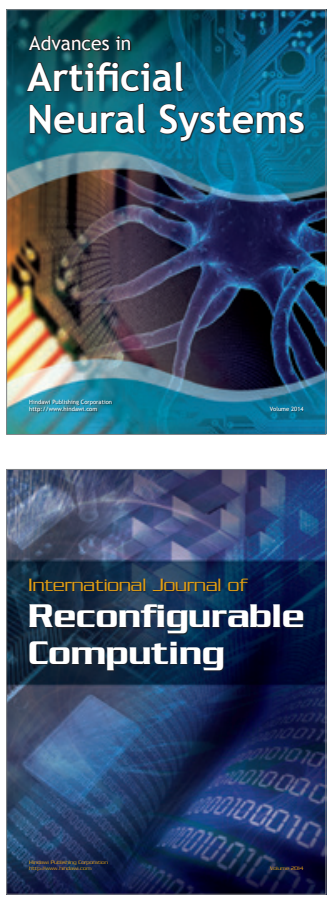
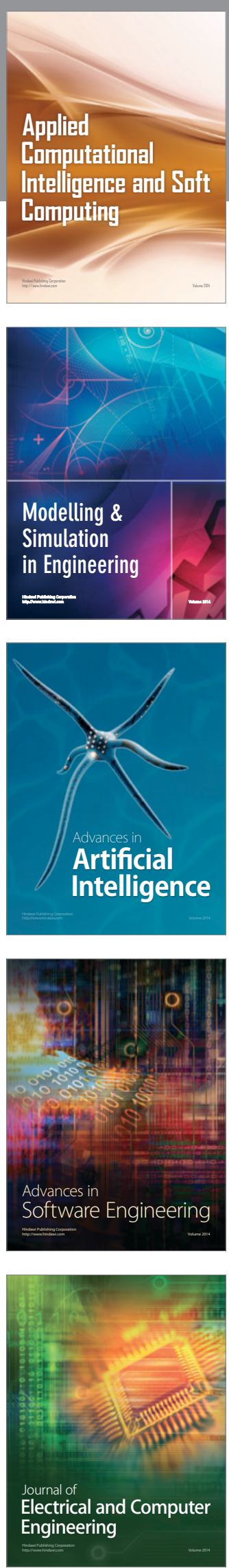